\title{
Estuarine ichthyofauna of the Paraguaçu River, Todos os Santos Bay, Bahia, Brazil
}

\author{
José Amorim Reis-Filho ${ }^{1,2,4}$, José de Anchieta Cintra da Costa Nunes ${ }^{1}$ \& Amanda Ferreira ${ }^{3}$ \\ 'Programa de Pós-graduação em Ecologia e Biomonitoramento, Universidade Federal da Bahia - UFBA, \\ Rua Barão de Geremoabo, s/n, Ondina, CEP 40170-000, Salvador, BA, Brasil \\ ${ }^{2}$ ICHTUS Soluções em Meio Ambiente Ltda, Rua Frederico Simões, Ed. Orlando Gomes, Sala 211, n. 513 , \\ Caminho das Árvores, CEP 41 820-774, Salvador, BA, Brasil, \\ e-mail: anchietaba@yahoo.com.br. \\ ${ }^{3}$ Instituto de Biologia, Universidade Federal Fluminense - UFF, Nitéroi, RJ, Brasil, \\ e-mail: amanda.rio.uff@gmail.com \\ ${ }^{4}$ Corresponding author: José Amorim Reis-Filho, e-mail: amorim_agua@yahoo.com.br
}

REIS-FILHO, J.A., NUNES, J.A.C.C. \& FERREIRA, A. Estuarine ichthyofauna of the Paraguaçu River, Todos os Santos Bay, Bahia, Brazil. Biota Neotrop. 10(4): http://www.biotaneotropica.org.br/v10n4/en/abstra ct?inventory+bn03610042010.

\begin{abstract}
Estuaries are areas of recognized importance in the development and sanctuaries of fishes and also a great source of fishing resources. This article presents an inventory of fishes from the Paraguaçu River estuary, Todos os Santos Bay, Bahia, Brazil. 28 points comprising the saline sections that may exist in a tropical estuary were sampled quarterly from March 2009 to February 2010 with the aid of bottom gill nets, surface gill nets, seine net and casting net. A total of 4.097 individuals were captured, belonging to 7 species of Chondrichthyes and 117 species of Actinopterygii (83 genera and 49 families). Most of the geographical extent of the estuary was sampled by assessing large spatial variability in fish assemblage structure. The curve of species accumulation (rarefaction) had stabilization with $1 / 2$ of the samples. The general structure of the assemblage is described and commented trough evidences for a new spatial organization of the fish fauna due to dam effects upstream the estuary.

Keywords: inventory, estuarine fishes, spatial distribution, effects upstream, brazilian northeast.
\end{abstract}

REIS-FILHO, J.A., NUNES, J.A.C.C. \& FERREIRA, A. Ictiofauna estuarina do Rio Paraguaçu, Baía de Todos os Santos, Bahia, Brasil. Biota Neotrop. 10(4): http://www.biotaneotropica.org.br/v10n4/pt/abstract?in ventory+bn03610042010.

Resumo: Estuários são áreas de reconhecida importância no desenvolvimento e refúgio dos peixes e também grande fonte de recursos pesqueiros. Este artigo apresenta um inventário dos peixes do estuário do Rio Paraguaçu, Baía de Todos os Santos, Bahia, Brasil. 28 pontos constituindo as três seções salinas que pode haver em um estuário tropical foram amostradas trimestralmente de março de 2009 a fevereiro de 2010 com auxílio de redes de espera de fundo, de superfície, redes de arrasto manual e tarrafas. Um total de 4.097 indivíduos foram capturados, pertencendo a 7 espécies de Chondrichthyes e 117 espécies de Actinopterygii (83 gêneros e 49 famílias). A maioria da extensão territorial do estuário foi amostrada para acessar maior variabilidade espacial na estrutura da assembléia de peixes. A curva de acumulação de espécies (rarefação) apresentou estabilização com $1 \frac{1}{2}$ das amostras. A estrutura geral da assembléia é descrita e comentada através de evidências para uma nova organização espacial da fauna de peixes devido a efeitos de barragem a montante do estuário.

Palavras-Chaves: inventário, peixes estuarinos, distribuição espacial, efeitos a montante, nordeste brasileiro. 


\section{Introduction}

The Paraguaçu River, the main tributary of the Todos os Santos Bay (BTS), is one of the most important aquatic systems of the Bahia State. This system is of high value for wildlife conservation and provides the main source of protein and income (i.e. consumption and commercialization of fish and shellfish) for the local communities (Barros et al. 2008). In spite of the ecological and economic importance of Paraguaçu River estuary $\left(127.9 \mathrm{~km}^{2}\right)$, this river is located on the west side of Todos os Santos Bay and its origin is related to the drowning of a fluvial valley, during MiddleHolocene, controlled by the system of faults from the western portion of Recôncavo Bay, especially for the Maragojipe Fault (Carvalho 2000). The estuary is composed by three sectors: the lower course of the river (with $16 \mathrm{~km}$ long and average depth of $1 \mathrm{~m}$ ), Iguape Bay (with $76.1 \mathrm{~km}^{2}$ and an intertidal area of $53.7 \%$ ) and by the Paraguaçu channel (with $18 \mathrm{~km}$ long and a average depth of $10 \mathrm{~m}$ ), which establishes a connection between the Iguape Bay and Todos os Santos Bay, associated with the good state of conservation and the presence of a wide mangrove area $\left(28 \mathrm{~km}^{2}\right)$, favoring a high fishing productivity and the presence of a wide riverside community involved with artisanal fishing (Genz et al. 2008). The municipality of Maragojipe, alongside the Iguape Bay, has come to be the second major fish producer in the Bahia State (BAHIAPESCA 1998), where in 2000 the IBAMA created the Iguape Bay Marine Extractive Reserve (Decree no number of 14/08/2000). During the 80's, the Pedra do Cavalo Dam (PCD) was built about $28 \mathrm{~km}$ from de mouth of Iguape Bay, causing changes on the hydrological regime of the river. In 2005, a new change occurred due to the implantation of the Pedra do Cavalo Hydroeletric Power Plant (HPP), changing the fresh water flow to energy generation (Genz et al. 2008). Nowadays there is a project in licensing for deployment of a port system within the estuary where extensive mangrove zone and estuarine sediment will be removed, setting one more impact scenario for the fish fauna as well as all the marine biota.

Few studies with the clear objective of surveying the fish fauna of the estuaries on Northeastern Brazil have been carried (Eskinazi 1970, 1972, Oliveira 1972, 1974, 1976, Araújo et al. 2000). Most studies focus on ecological aspects of ichthyofauna from shallow areas (Alves \& Soares Filho 1996, Castro 2001, Barletta et al. 2003, Carvalho Neta \& Castro 2008, Paiva et al. 2008, Reis-Filho et al. 2010), fishes as accompanying fauna in shrimp fisheries (Barros \& Jonsson 1967, Albuquerque 1994, Santos et al. 1998, Santos 2000) and few with deep drags targeted for capture of fishes (Barletta et al. 2005). Thus, studies that seek access the fish fauna evaluating the variability of habitats of the estuarine system are missing.

In the Paraguaçu River estuary there is only one study approaching communities of fish in the mouth (Reis-Filho, in press), and a work addressing expansion of geographical distribution (Santos et al. 2009). Studies of faunal surveys constitute the initial step for biological studies and management of an area, by providing basic information on the composition and structure of the investigated fauna (Casatti et al. 2001). However, the fact of the Todos os Santos Bay being the second largest semi-enclosed bay in Brazil and have no ichthyofaunal study in their estuaries (Andrade-Tubino et al. 2008), besides the fact that studies reported for Brazilian estuaries are credited only to the north and south region (Faunce \& Serafy 2006) sets up such a worrying scenario. The urgent need to know the ichthyofauna of the place so as to develop conservation and management measures corroborates the lack of these studies in estuarine environments. This way, the estuarine ichthyofauna from Paraguaçu River, Todos os Santos Bay, Bahia was studied the main factors that influence the distribution and composition, by making a parallel with the effect of dam upstream the estuary.

\section{Material and Methods}

Fishes were collected at 28 points within the Paraguaçu River estuary with quarterly samples between March 2009 and February 2010 (Figure 1). Several types of collectors were used: for marginal environments and with depths up to $3 \mathrm{~m}$, trawl manual $(12 \mathrm{~m} \times 2 \mathrm{~m}$ with $12 \mathrm{~mm}$ mesh), gillnets ( $100 \mathrm{~m} \times 3 \mathrm{~m}$ with $15 \mathrm{~mm}$ mesh) and flue (casting net ) (10 mm mesh). For environments with depth between 4 and $20 \mathrm{~m}$, bottom gill nets $(300 \mathrm{~m} \times 3 \mathrm{~m}$ with $20 \mathrm{~mm}, 30 \mathrm{~mm}$, $40 \mathrm{~mm}, 50 \mathrm{~mm}$ and $60 \mathrm{~mm}$ mesh) and surface gill nets $(200 \mathrm{~m} \times 4 \mathrm{~m}$ with $15 \mathrm{~mm}$ mesh) were used. In order to evaluate the ichthyofauna distribution in the estuary, this one was segmented into three sections (lower, middle and upper).

Specimens were transported in ice and stored frozen until identification with the most current literature (Figueiredo \& Menezes 1978, 1980, 2000, Menezes \& Figueiredo 1980, 1985, Carpenter 2002a, b, Marceniuk 2005; Moura \& Lindeman 2007). Total length $(\mathrm{mm})$ and weight $(\mathrm{g})$ was recorded for each specimen. Vouchers were deposited at the Universidade Federal da Bahia (UFBA). The occurrence of constancy of species was calculated as function of the number of times each species toke place in relation to the total collections done (Dajoz 1973). In order to identify the dominant species, we used the Relative Importance Index (IRI) as defined by Beaumord (1991), where $\mathrm{Ni}$ is the number of species individuous, $\mathrm{Wi}$ is the weight of the specimens of the species and $\mathrm{C}$ is the constancy of occurrence (Figure 2). The efficiency of the inventory was assessed by the method of species accumulation (rarefaction) at program PAST® (Paleontological Statistics, ver. 1.75). A multivariate analysis of type DCA (Detrended Correspondence Analysis) (ter Braak 1995) was applied to the data of species abundance dominant and constant, transformed into neperian logarithm to synthesize community variation along the spatial gradient. The salinity was measured at all points during the sampling campaigns with the aid of optical refractometer in order to define the different saline zones within the estuary.

The fish species were classified in five estuarine-use functional groups proposed by Elliott et al. (2007): 1) marine stragglers, species that spawn at sea and typically enter estuaries only in low numbers most frequently in the lower reaches and occur where salinities are c. 35 ;2) marine migrants, species that spawn at sea and often enter estuaries in large numbers, particularly as juveniles. Some of these species are highly euryhaline and move throughout the full length of the estuary; 3 ) estuarine residents, estuarine species capable of completing their entire life cycle within the estuary environment; 4) estuarine migrants, estuarine species that have larval stages of their life cycles completed outside the estuary or are also represented by discrete marine or freshwater populations; and 5) semi-catadromous, species whose spawning run extends only to estuarine areas rather than the marine environment. The scientific nomenclature followed Nelson (1994), Eschmeyer (2006), Froese \& Pauly (2006), Marceniuk \& Menezes (2007), Marceniuk (2005) and Menezes et al. (2003). Classification according to habitat use was compared with species listed on the work of Barletta-Bergan (1999), Camargo (1999), Barletta et al. (2003, 2005, 2008), Fisher et al. (2004) and Moraes et al. (2009).

\section{Results}

A slight salt gradient was observed in the Paraguaçu estuary, with values above 35 near the mouth dropping to 27 in almost all the middle estuary (Iguape Bay). From this area the salinity has values increasingly smaller until it reaches 0 (zero), where the upper estuary starts. In this last sector, the salinity ranged from 0 to 5 as the movement of tides. The estuary of the Paraguaçu River during the sampling campaigns showed a behavior predominantly saline. 


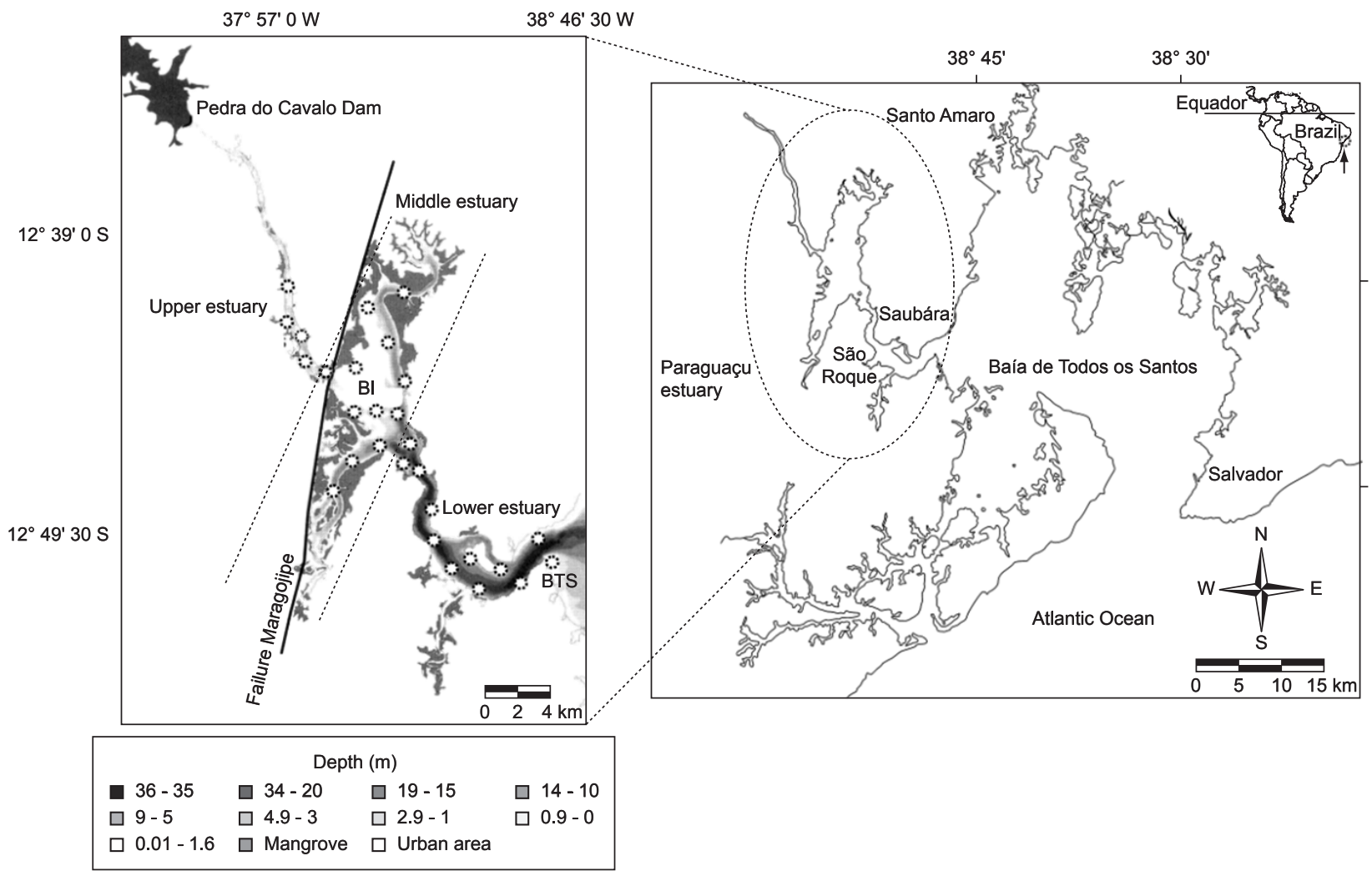

Figure 1. Location of Paraguaçu River estuary with sampling points. IB: Iguape Bay. (Adepted of Genz et al. 2008).

Figura 1. Localização do estuário do Rio Paraguaçu com pontos amostrados. IB: Baía de Iguape. (Adaptado de Genz et al. 2008).

$$
\mathrm{IRI}=\frac{(\mathrm{NiWiC})}{\sum(\mathrm{NiWiC})} \times 100
$$

Figure 2. Formula used to calculate the Index of Relative Importance.

Figura 2. Fórmula usada para calcular o Índice de Importância Relativa.

The species accumulation curve, obtained by the technique of rarefaction of samples indicates that the samples with $1 / 2$ the number of species seems to reach a plateau-linear with most species being accessed in these catches (Figure 3). A total of 224 samples were taken and 4.097 specimens were captured, belonging to 7 species of Chondrichthyes and 117 species of Actinopterygii (83 genera and 49 families). Most recorded species (62\%) are definitely marine fishes, but a considerable number of estuarine species was also recorded (35\%). Four Actinopterygii were identified only at the level of genus (Anchoa sp., Paralichthys sp., Stellifer sp. and Mugil sp.). Perciformes (IRI $=61.5 \%$ ) was the most important order, followed by Clupeiformes (IRI $=18.2 \%)$, Pleuronectiformes $(I R I=11.2 \%)$ and Tetraodontiformes $(7.9 \%)$. Sciaenidae e Carangidae were the most abundant families in number of species (12 each). Sciaenidae showed the most weight (39.8\%). Sciaenidae and Gobiidae were the most abundant families in number of individuals (14.8\% and $11.9 \%$, respectively), followed to Gerreidae (9.4\%), Engraulidae (8.2\%), Clupeidae (5.5\%) and Tetraodontidae (5.3\%), together they accounted more than $55 \%$ of total abundance (Table 1). Among the nine most abundant and constant species, have been two of sciaenids, Stelifer rastrifer (Jordan, 1889) and Cynoscion microlepidotus (Cuvier, 1830) dominating the middle estuary, two of gerreids Diapterus rhombeus (Cuvier, 1829) and Eucinostomus argenteus Baird \& Girard, 1855 abundant and frequent in the lower and middle estuary, one of Tetraodontidae, Sphoeroides greeleyi Gilbert, 1900 abundant and constant in all three sectors and one Pristigasteridae, Pellona harroweri (Fowler, 1917) abundant and constant in the lower and middle estuary, and these were responsible for $52.5 \%$ of the expressed dominance (IRI).

It was possible to identify assemblages with distinct patterns of habitat use according to the zones of depths, behavior in the estuary and salt sectors. The dispersal of species along the axis 1 of the DCA, held in the Paraguaçu River estuary, indicates that the species exclusively caught in shallow areas have high abundances in all saline sectors of the estuary and the species caught in deep water are more abundant in the middle estuary (Figure 4a). Yet, the resident estuarine species show preference for the middle estuary with higher abundances recorded in this sector and reduced catches in the lower estuary. The marine migrants were found in all sectors and marine stragglers were from the mouth to the middle estuary with homogeneous densities among sectors (Figure 4b).

\section{Discussion}

The trend in stabilization of the species accumulation curve with about $1 / 2$ of the samples corroborates with the information that in the estuarine environment the fish assemblage is dominated by few species (Day et al. 1989, Santos et al. 2002, Godefroid et al. 2004) and its basic structure may appear relatively stable or even predictable (Blaber \& Blaber 1980, Whitfield 1999, Paterson \& Whitfield 2000). One positive point for the stabilization of the accumulation curve was the use of various sampling techniques that have covered a great variety of habitats on the spatial gradient (e.g. shallow and deep zones, tidal channels and tidal flats). Among other aspects, the limited use of sample artifacts, especially towed passive network (gillnets) on the American continent, are responsible for poor knowledge about the habitat use by estuarine fish fauna (Faunce \& Serafy 2006). 
Table 1. Checklist of fish species captured at the Paraguaçu River estuary, Bahia, Brazil. $\mathrm{N}=$ Total Number of individuals; $\mathrm{N}(\%)=$ Relative abundance; $\mathrm{W}(\%)=$ Relative weight; $\mathrm{C}=$ Occurrence constancy; IRI = Importance relative index; HU = Habitat use; MS = Marine stragglers; ES = Estuarine residents; $\mathrm{MM}=$ Marine migrants; $\mathrm{EM}=$ Estuarine Migrants; and $\mathrm{SC}=$ Semi-catadroms. $*=$ marine species found in the middle estuary.

Tabela 1. Lista das espécies de peixes capturadas no estuário do Rio Paraguaçu, Bahia, Brazil. $\mathrm{N}=$ Número total de indivíduos; $\mathrm{N}(\%)=\mathrm{Abundância} \mathrm{relativa;}$ $\mathrm{W}(\%)=$ Peso relativo; $\mathrm{C}=$ Constância de Ocorrência; IRI = Índice de importância relativa; HU = Uso do habitat; MS = Visitantes Marinhos; ES = Estuarinos residentes; $\mathrm{MM}=$ Migrantes Marinhos; EM = Migrantes Estuarinos; e SC = Semi-catádromos. * = espécies marinhas encontradas no estuário médio.

\begin{tabular}{|c|c|c|c|c|c|c|}
\hline Class, Order, Family and Species & $\mathbf{N}$ & $\mathbf{N}(\%)$ & $\mathrm{W}(\%)$ & $\mathrm{C}(\%)$ & IRI & HU \\
\hline \multicolumn{7}{|l|}{ Class Chodrichthyes } \\
\hline \multicolumn{7}{|l|}{ Order Charchariniformes } \\
\hline \multicolumn{7}{|l|}{ CHACHARINIDAE } \\
\hline Rhizoprionodon porosus (Poey, 1861) & 4 & 0.09 & 0.07 & 0.8 & 0.003 & MS \\
\hline \multicolumn{7}{|l|}{ Order Rajiformes } \\
\hline \multicolumn{7}{|l|}{ NARCINIDAE } \\
\hline Narcine brasiliensis (Olfers, 1831) & 2 & 0.04 & 0.06 & 0.8 & 0.002 & MS \\
\hline \multicolumn{7}{|l|}{ RHINOBATIDAE } \\
\hline Rhinobatos percellens (Walbaum, 1792) & 4 & 0.09 & 0.1 & 0.8 & 0.009 & MS \\
\hline \multicolumn{7}{|l|}{ DASYATIDAE } \\
\hline Dasyatis guttata (Bloch \& Schneider, 1801) & 2 & 0.04 & 1.2 & 0.8 & 0.01 & MS \\
\hline Dasyatis americana (Hildebrand \& Schroeder, 1928) & 3 & 0.07 & 1.3 & 0.8 & 0.02 & MS* \\
\hline \multicolumn{7}{|l|}{ GYMNURIDAE } \\
\hline Gymnura micrura (Bloch \& Schneider, 1801) & 1 & 0.02 & 0.9 & 0.4 & 0.01 & MS* \\
\hline \multicolumn{7}{|l|}{ MYLIOBATIDAE } \\
\hline Rhinoptera bonasus (Mitchill, 1815) & 1 & 0.02 & 0.8 & 0.4 & 0.01 & MS* \\
\hline \multicolumn{7}{|l|}{ Class Actinopterygii } \\
\hline \multicolumn{7}{|l|}{ Order Anguliformes } \\
\hline \multicolumn{7}{|l|}{ MURAENIDAE } \\
\hline Gymnothorax ocellatus Agassiz, 1831 & 1 & 0.02 & 0.008 & 0.4 & 0.001 & MS \\
\hline Gymnothorax moringa (Cuvier, 1829) & 3 & 0.07 & 1.1 & 0.8 & 0.02 & MM \\
\hline \multicolumn{7}{|l|}{ Order Albuliformes } \\
\hline \multicolumn{7}{|l|}{ ALBULIDAE } \\
\hline Albula vulpes (Linnaeus, 1758) & 78 & 1.94 & 0.9 & 5.9 & 0.3 & $\mathrm{EM}$ \\
\hline \multicolumn{7}{|l|}{ Order Clupeiformes } \\
\hline \multicolumn{7}{|l|}{ ENGRAULIDAE } \\
\hline Anchoa af. januaria Hildebrand, 1943 & 44 & 0.84 & 0.7 & 4.7 & 0.2 & ER \\
\hline Anchoa lyolepis (Evermann \& Marsh, 1900) & 55 & 1.12 & 0.9 & 4.3 & 0.2 & ER \\
\hline Anchoa spinifer (Valenciennes, 1848) (MZUFBA 5619) & 48 & 1.19 & 0.7 & 3.5 & 0.2 & ER \\
\hline Anchoa sp. & 12 & 0.30 & 0.2 & 2.3 & 0.05 & ER \\
\hline Anchovia clupeoides (Swainson, 1839) & 42 & 0.55 & 0.9 & 10.1 & 0.9 & $M M^{*}$ \\
\hline Cetengraulis edentulus (Cuvier, 1829) (MZUFBA 5715 and 5951) & 105 & 2.50 & 1.98 & 10.4 & 1.1 & EM \\
\hline Lycengraulis grossidens (Agassiz, 1829) & 32 & 0.79 & 0.8 & 5.4 & 0.8 & MS \\
\hline \multicolumn{7}{|l|}{ PRISTIGASTERIDAE } \\
\hline Pellona harroweri (Fowler, 1917) & 111 & 3.75 & 4.38 & 32.4 & 3.2 & MS \\
\hline \multicolumn{7}{|l|}{ CLUPEIDAE } \\
\hline Lile piquitinga (Schreiner \& Miranda Ribeiro, 1903) (MZUFBA 5601) & 66 & 1.64 & 0.5 & 16.7 & 1.6 & ER \\
\hline Opisthonema oglinum (Lesueur, 1818) & 109 & 2.71 & 2.6 & 34.5 & 1.5 & $M M^{*}$ \\
\hline Harengula clupeola (Cuvier, 1829) & 54 & 1.34 & 1.2 & 15.6 & 0.5 & MS* \\
\hline \multicolumn{7}{|l|}{ Order Siluriformes } \\
\hline \multicolumn{7}{|l|}{ ARIIDAE } \\
\hline Sciades herzbergii (Bloch, 1794) & 78 & 1.94 & 9.8 & 23.5 & 2.4 & ER \\
\hline Sciades couma (Valenciennes, 1840) & 13 & 0.32 & 2.3 & 4.6 & 1.4 & ER \\
\hline \multicolumn{7}{|l|}{ Order Aulopiformes } \\
\hline \multicolumn{7}{|l|}{ SYNODONTIDAE } \\
\hline Synodus foetens (Linnaeus, 1766) (MZUFBA 5721) & 21 & 0.52 & 0.4 & 6.8 & 0.2 & MS \\
\hline
\end{tabular}


Table 1. Continued...

\begin{tabular}{|c|c|c|c|c|c|c|}
\hline Class, Order, Family and Species & $\mathbf{N}$ & $\mathbf{N}(\%)$ & $\mathrm{W}(\%)$ & $\mathrm{C}(\%)$ & IRI & HU \\
\hline \multicolumn{7}{|l|}{ Order Batrachoidiformes } \\
\hline \multicolumn{7}{|l|}{ BATRACHOIDIDAE } \\
\hline Amphichthys cryptocentrus (Valenciennes, 1837) & 6 & 0.15 & 0.7 & 2.2 & 0.04 & ER \\
\hline Thalassophryne nattereri Steindachner, 1876 & 15 & 0.37 & 0.6 & 4.1 & 0.07 & MM \\
\hline \multicolumn{7}{|l|}{ OGCOCEPHALIDAE } \\
\hline Ogcocephalus vespertilio (Linnaeus, 1758) & 19 & 0.47 & 0.9 & 3.5 & 0.06 & MM \\
\hline \multicolumn{7}{|l|}{ Order Mugiliformes } \\
\hline \multicolumn{7}{|l|}{ MUGILIDAE } \\
\hline Mugil curema Valenciennes, 1836 & 76 & 1.89 & 2.3 & 15.7 & 1.5 & EM \\
\hline Mugil liza Valenciennes, 1836 & 32 & 0.79 & 1.2 & 7.6 & 0.8 & EM \\
\hline Mugil sp. & 8 & 0.20 & 0.04 & 2.3 & 0.05 & EM \\
\hline \multicolumn{7}{|l|}{ Order Atheriniformes } \\
\hline \multicolumn{7}{|l|}{ ATHERINOPSIDAE } \\
\hline $\begin{array}{l}\text { Atherinella brasiliensis (Quoy \& Gaimard, 1825) (MZUFBA 5917, 5921, } \\
5944 \text { and 5952) }\end{array}$ & 167 & 4.14 & 1.9 & 39.6 & 3.4 & ER \\
\hline \multicolumn{7}{|l|}{ Order Beloniformes } \\
\hline \multicolumn{7}{|l|}{ BELONIDAE } \\
\hline Strongylura timиси (Walbaum, 1792) & 5 & 0.12 & 0.14 & 0.5 & 0.003 & MS \\
\hline Strongylura marina (Walbaum, 1792) & 3 & 0.07 & 0.1 & 0.5 & 0.002 & MS \\
\hline \multicolumn{7}{|l|}{ HEMIRAMPHIDAE } \\
\hline Hyporhamphus unifasciatus (Ranzani, 1841) (MZUFBA 5593) & 17 & 0.42 & 0.05 & 0.4 & 0.01 & MS \\
\hline \multicolumn{7}{|l|}{ Order Gasterosteiformes } \\
\hline \multicolumn{7}{|l|}{ SYNGNATHIDAE } \\
\hline Syngnathus sp. & 3 & 0.07 & 0.01 & 1.3 & 0.01 & MS \\
\hline Hippocampus reidi Ginsburg, 1933 & 6 & 0.15 & 0.03 & 1.3 & 0.02 & ER \\
\hline \multicolumn{7}{|l|}{ FISTULARIDAE } \\
\hline Fistularia tabacaria Linnaeus, 1758 & 18 & 0.45 & 0.09 & 3.1 & 0.9 & MS* \\
\hline \multicolumn{7}{|l|}{ Order Scorpaeniformes } \\
\hline \multicolumn{7}{|l|}{ DACTYLOPTERIDAE } \\
\hline \multirow[t]{2}{*}{ Dactylopterus volitans (Linnaeus, 1758) (MZUFBA 6271) } & 33 & 0.82 & 1.1 & 5.1 & 1.2 & \\
\hline & & & & & & $\mathrm{MM}^{*}$ \\
\hline \multicolumn{7}{|l|}{ SCORPAENIDAE } \\
\hline Scorpaena plumieri Bloch, 1789 & 5 & 0.12 & 0.04 & 1.3 & 0.004 & MS \\
\hline \multicolumn{7}{|l|}{ TRIGLIDAE } \\
\hline $\begin{array}{l}\text { Prionotus punctatus (Bloch, 1793) (MZUFBA 5675, 5680, 5688, 5709, 5712, } \\
\text { 5717, 6201, } 6207 \text { and 6223) }\end{array}$ & 23 & 0.57 & 0.9 & 3.5 & 0.8 & $\mathrm{MM}^{*}$ \\
\hline \multicolumn{7}{|l|}{ Order Perciformes } \\
\hline \multicolumn{7}{|l|}{ MULLIDAE } \\
\hline Pseudupeneus maculatus (Bloch, 1793) & 6 & 0.15 & 0.03 & 2.3 & 0.01 & MS \\
\hline \multicolumn{7}{|l|}{ CENTROPOMIDAE } \\
\hline Centropomus undecimalis (Bloch, 1792) (MZUFBA 5611, 5596 and 5695) & 28 & 0.69 & 1.6 & 6.7 & 1.4 & ER \\
\hline Centropomus parallelus Poey, 1860 & 15 & 0.37 & 0.9 & 3.6 & 1.3 & ER \\
\hline SERRANIDAE & & & & & & \\
\hline Diplectrum radiale (Quoy \& Gaimard, 1824) (MZUFBA 5600) & 21 & 0.52 & 0.04 & 4.8 & 0.04 & $\mathrm{MM}^{*}$ \\
\hline Serranus flaviventris (Cuvier, 1829) (MZUFBA 6225) & 19 & 0.47 & 0.01 & 5.6 & 0.01 & $\mathrm{MM}^{*}$ \\
\hline EPINEPHILIDAE & & & & & & \\
\hline Rypticus randalli Couternay, 1967 (MZUFBA 5607 and 6224) & 58 & 1.44 & 0.4 & 7.5 & 0.07 & MS* \\
\hline OPISTOGNATHIDAE & & & & & & \\
\hline Opistognathus cuvieri Valenciennes, 1836 & 3 & 0.07 & 0.01 & 1.3 & 0.01 & MS \\
\hline CARANGIDAE & & & & & & \\
\hline Oligoplites palometa (Cuvier, 1832) & 19 & 0.47 & 0.7 & 7.8 & 0.09 & MM \\
\hline
\end{tabular}


Table 1. Continued...

\begin{tabular}{|c|c|c|c|c|c|c|}
\hline Class, Order, Family and Species & $\mathbf{N}$ & $\mathbf{N}(\%)$ & $\mathrm{W}(\%)$ & $\mathrm{C}(\%)$ & IRI & HU \\
\hline Oligoplites saliens (Bloch, 1793) & 21 & 0.52 & 0.9 & 8.9 & 0.9 & MM \\
\hline Oligoplites saurus (Bloch \& Schneider, 1801) & 36 & 0.89 & 1.2 & 13.4 & 1.1 & MM \\
\hline Oligoplites sp. & 3 & 0.07 & 0.08 & 0.4 & 0.04 & MM \\
\hline Carangoides crysos (Mitchill, 1815) & 10 & 0.25 & 0.09 & 2.3 & 0.05 & MM \\
\hline Carangoides bartholomaei (MZUFBA 5589) & 15 & 0.37 & 0.1 & 4.5 & 0.06 & MM \\
\hline Caranx latus Agassiz, 1831 (MZUFBA 5590 and 5606) & 78 & 1.94 & 3.45 & 37.8 & 2.6 & $\mathrm{MM}^{*}$ \\
\hline Caranx hippos (Linnaeus, 1766) & 6 & 0.15 & 0.7 & 2.3 & 0.09 & MS \\
\hline Chloroscombrus chrysurus (Linnaeus, 1766) & 66 & 1.64 & 2.34 & 25.7 & 2.4 & MS* \\
\hline Selene setapinnis (Mitchill, 1815) & 4 & 0.10 & 0.03 & 0.4 & 0.03 & MS \\
\hline Selene vomer (Linnaeus, 1758) & 11 & 0.27 & 0.5 & 5.6 & 0.4 & MS \\
\hline Trachinotus falcatus (Linnaeus, 1758) (MZUFBA 5594) & 7 & 0.17 & 0.3 & 4.5 & 0.3 & MS \\
\hline \multicolumn{7}{|l|}{ LUTJANIDAE } \\
\hline Lutjanus jocu (Bloch \& Schneider, 1801) & 12 & 0.30 & 0.7 & 5.6 & 0.4 & MM \\
\hline Lutjanus alexandrei (Moura \& Lindeman, 2007) & 7 & 0.17 & 0.5 & 3.5 & 0.05 & MM \\
\hline Lutjanus synagris (Linnaeus, 1758) (MZUFBA 5602 and 5942) & 43 & 1.07 & 1.2 & 19.6 & 0.9 & $\mathrm{MM}^{*}$ \\
\hline \multicolumn{7}{|l|}{ LOBOTIDAE } \\
\hline Lobotes surinamensis (Bloch, 1790) & 7 & 0.17 & 0.6 & 5.9 & 0.02 & MM \\
\hline \multicolumn{7}{|l|}{ GERREIDAE } \\
\hline Diapterus rhombeus (Cuvier, 1829) & 117 & 2.90 & 1.8 & 28.5 & 2.5 & ER \\
\hline Diapterus auratus Ranzani, 1842 & 38 & 0.94 & 0.4 & 25.7 & 0.6 & ER \\
\hline $\begin{array}{l}\text { Eucinostomus gula (Quoy \& Gaimard, 1824) (MZUFBA 5595, 5605, 5671, } \\
\text { 5702, } 5704 \text { and } 6218 \text { ) }\end{array}$ & 46 & 1.14 & 0.5 & 26.7 & 0.9 & MM \\
\hline $\begin{array}{l}\text { Eucinostomus argenteus Baird \& Girard, } 1855 \text { (MZUFBA 5599, 5684, 5685, } \\
5700,5701,5703 \text { and 6229) }\end{array}$ & 111 & 2.76 & 0.6 & 38.8 & 2.4 & ER \\
\hline $\begin{array}{l}\text { Eucinostomus melanopterus (Bleeker, 1863) (MZUFBA 5592, 5597, } 5705 \\
\text { and 6227) }\end{array}$ & 48 & 1.19 & 1 & 20.8 & 0.09 & $\mathrm{MM}^{*}$ \\
\hline Eugerres brasilianus Cuvier, 1830 ) (MZUFBA 5614) & 16 & 0.40 & 0.07 & 3.4 & 0.06 & MM \\
\hline Gerres cinereus (Walbaum, 1792) & 11 & 0.27 & 0.04 & 2.3 & 0.005 & $\mathrm{MM}^{*}$ \\
\hline \multicolumn{7}{|l|}{ HAEMULIDAE } \\
\hline Haemulon steindachneri (Jordan \& Gilbert, 1882) (MZUFBA 5608 and 5722) & 59 & 1.40 & 0.8 & 4.5 & 0.09 & MM \\
\hline Haemulon squamipinna Rocha \& Rosa, 1999 & 7 & 0.17 & 0.03 & 1.3 & 0.002 & MS \\
\hline Haemulon parra (Desmarest, 1823) & 9 & 0.22 & 0.05 & 1.3 & 0.003 & MS \\
\hline Pomadasys corvinaeformis (Steindachner, 1868) & 7 & 0.17 & 0.007 & 1.3 & 0.002 & MS \\
\hline Genyatremus luteus (Bloch, 1795) (MZUFBA 5716) & 19 & 0.47 & 0.009 & 2.3 & 0.01 & ER \\
\hline Anisotremus virginicus (Linnaeus, 1758) & 2 & 0.05 & 0.002 & 0.4 & 0.001 & MM \\
\hline Anisotremus surinamensis & 7 & 0.17 & 0.003 & 1.3 & 0.002 & $\mathrm{MM}^{*}$ \\
\hline \multicolumn{7}{|l|}{ SPARIDAE } \\
\hline Archosargus rhomboidalis (Linnaeus, 1758) & 9 & 0.22 & 0.007 & 1.3 & 0.002 & MM \\
\hline \multicolumn{7}{|l|}{ POLYNEMIDAE } \\
\hline Polydactylus virginicus (Linnaeus, 1758) & 67 & 1.11 & 2.11 & 33.7 & 2.3 & MS \\
\hline \multicolumn{7}{|l|}{ SCIAENIDAE } \\
\hline Cynoscion jamaicensis (Vaillant \& Bocourt, 1883) & 3 & 0.07 & 0.04 & 0.8 & 0.001 & EM \\
\hline Cynoscion microlepidotus (Cuvier, 1830) & 122 & 3.03 & 6.77 & 44.7 & 5.6 & ER \\
\hline Cynoscion acoupa (Lacepède, 1801) & 23 & 0.57 & 0.95 & 5.6 & 0.02 & ER \\
\hline Cynoscion leiarchus (Cuvier, 1830) & 74 & 1.84 & 0.9 & 10.9 & 2.9 & EM \\
\hline Isopisthus parvipinnis (Cuvier, 1830) & 33 & 0.77 & 4.11 & 27.8 & 2.4 & $\mathrm{MM}^{*}$ \\
\hline Larimus breviceps Cuvier, 1830 & 2 & 0.05 & 0.002 & 0.4 & 0.001 & EM \\
\hline Macrodon ancylodon (Bloch \& Schneider, 1801) & 34 & 0.75 & 3.98 & 29.9 & 3.6 & $\mathrm{MM}^{*}$ \\
\hline Menticirrhus americanus (Linnaeus, 1758) & 5 & 0.12 & 0.05 & 1.3 & 0.003 & ER \\
\hline Micropogonias furnieri (Desmarest, 1823) & 34 & 0.84 & 0.9 & 2.1 & 0.03 & EM \\
\hline Stellifer sp. & 3 & 0.07 & 0.009 & 0.8 & 0.003 & ER \\
\hline
\end{tabular}


Table 1. Continued..

\begin{tabular}{|c|c|c|c|c|c|c|}
\hline Class, Order, Family and Species & $\mathbf{N}$ & $\mathbf{N}(\%)$ & $\mathrm{W}(\%)$ & $\mathrm{C}(\%)$ & IRI & HU \\
\hline Stellifer rastrifer (Jordan, 1889) (MZUFBA 5718) & 178 & 4.42 & 7.89 & 59.9 & 15.5 & ER \\
\hline Stellifer stellifer (Bloch, 1790) & 99 & 2.46 & 0.9 & 9.9 & 0.06 & ER \\
\hline \multicolumn{7}{|l|}{ GOBIIDAE } \\
\hline Bathygobius soporator (Valenciennes, 1837) (MZUFBA 5615) & 49 & 1.22 & 0.21 & 5.6 & 0.05 & ER \\
\hline Gobionellus oceanicus (Pallas, 1770) & 43 & 1.07 & 0.2 & 5.1 & 0.04 & ER \\
\hline Ctenogobius stomatus Starks, 1913 & 135 & 3.35 & 1.04 & 50.4 & 11.7 & ER \\
\hline Ctenogobius boleosoma (Jordan \& Gilbert, 1882) & 57 & 1.41 & 0.2 & 5.6 & 0.06 & ER \\
\hline Ctenogobius stigmaticus (Poey, 1860) & 158 & 3.92 & 1.08 & 55.7 & 10.1 & ER \\
\hline Ctenogobius smaragdus (Valenciennes, 1837) & 45 & 1.12 & 0.15 & 12.3 & 0.05 & ER \\
\hline Microgobius meeki Evermann \& Marsh, 1900 & 4 & 0.10 & 0.001 & 1.3 & 0.002 & ER \\
\hline \multicolumn{7}{|l|}{ EPHIPPIDAE } \\
\hline Chaetodipterus faber (Broussonet, 1782) (MZUFBA 5603) & 28 & 0.69 & 0.1 & & 0.009 & MM \\
\hline \multicolumn{7}{|l|}{ ACANTHURIDAE } \\
\hline Acanthurus bahianus Castelnau, 1855 & 2 & 0.05 & 0.004 & 0.4 & 0.001 & MS \\
\hline \multicolumn{7}{|l|}{ SPHYRAENIDAE } \\
\hline Sphyraena barracuda (Edwards, 1771) & 9 & 0.22 & 0.09 & 1.3 & 0.004 & MM \\
\hline Sphyraena guachancho Cuvier, 1829 & 3 & 0.07 & 0.04 & 0.4 & 0.001 & MM \\
\hline \multicolumn{7}{|l|}{ TRICHIURIDAE } \\
\hline Trichiurus lepturus Linnaeus, 1758 & 32 & 0.79 & 0.1 & 2.1 & 0.05 & EM \\
\hline \multicolumn{7}{|l|}{ SCOMBRIDAE } \\
\hline Scomberomorus brasiliensis Collete, Russo \& Zavala-Camin, 1978 & 3 & 0.07 & 0.003 & 0.8 & 0.002 & MS \\
\hline \multicolumn{7}{|l|}{ Order Pleuronectiformes } \\
\hline \multicolumn{7}{|l|}{ PARALICHTHYDAE } \\
\hline Paralichthys brasiliensis (Ranzani, 1842) & 8 & 0.20 & 0.04 & 1.8 & 0.007 & MM \\
\hline Paralichthys tropicus Ginsburg, 1933 & 2 & 0.05 & 0.001 & 0.4 & 0.001 & MM \\
\hline Paralichthys sp. & 2 & 0.05 & 0.001 & 0.4 & 0.001 & MM \\
\hline Citharichthys spilopterus Günther, 1862 & 65 & 1.61 & 0.15 & 3.4 & 0.08 & ER \\
\hline Etropus crossotus Jordan \& Gilbert, 1882 (MZUFBA 5609 and 5616) & 44 & 1.09 & 0.13 & 2.5 & 0.06 & ER \\
\hline Syacium micrurum Ranzani, 1842 & 2 & 0.05 & 0.002 & 0.4 & 0.001 & MM \\
\hline \multicolumn{7}{|l|}{ ACHIRIDAE } \\
\hline Achirus lineatus (Linnaeus, 1758) (MZUFBA 5620) & 31 & 0.77 & 0.15 & 13.4 & 0.1 & ER \\
\hline Achirus declives Chabanaud, 1940 & 12 & 0.30 & 0.09 & 2.4 & 0.01 & ER \\
\hline Trinectes paulistanus (Miranda Ribeiro, 1915) & 54 & 1.34 & 0.14 & 22.9 & 0.3 & ER \\
\hline Trinectes microphthalmus (Chabanaud, 1928) & 4 & 0.10 & 0.01 & 0.8 & 0.003 & MM \\
\hline \multicolumn{7}{|l|}{ CYNOGLOSSIDAE } \\
\hline Symphurus plagusia (Bloch \& Schneider, 1801) & 13 & 0.32 & 0.06 & 4.5 & 0.008 & MM \\
\hline Symphurus diomedianus (Goode \& Bean, 1885) & 2 & 0.05 & 0.001 & 0.8 & 0.001 & MS \\
\hline \multicolumn{7}{|l|}{ Order Tetraodontiformes } \\
\hline \multicolumn{7}{|l|}{ MONOCANTHIDAE } \\
\hline Stephanolepis setifer (Bennett, 1831) (MZUFBA 5612) & 7 & 0.17 & 0.009 & 1.3 & 0.005 & MS \\
\hline Aluterus heudelotii Hollard, 1855 & 3 & 0.07 & 0.002 & 0.4 & 0.001 & MS \\
\hline \multicolumn{7}{|l|}{ TETRAODONTIDAE } \\
\hline $\begin{array}{l}\text { Sphoeroides testudineus (Linnaeus, 1758) (MZUFBA 5672, 5673, 5674, 5678, } \\
\text { 5679, 5682, 5683, } 5687 \text { and 5696) }\end{array}$ & 76 & 1.89 & 0.9 & 23.5 & 0.09 & ER \\
\hline $\begin{array}{l}\text { Sphoeroides greeleyi Gilbert, } 1900 \text { (MZUFBA 5916, 5618, 5676, 5677, 5681, } \\
5686 \text { and 5706) }\end{array}$ & 118 & 2.80 & 1.4 & 49.8 & 1.5 & $\mathrm{MM}^{*}$ \\
\hline Sphoeroides spengleri (Bloch, 1785) & 17 & 0.42 & 0.05 & 5.8 & 0.01 & MM \\
\hline Achantostracium quadricornis (Linnaeus, 1758) & 7 & 0.17 & 0.001 & 4.5 & 0.006 & MS \\
\hline \multicolumn{7}{|l|}{ DIODONTIDAE } \\
\hline Chilomycterus spinosus (Linnaeus, 1758) & 59 & 1.23 & 0.09 & 38.6 & 0.05 & MS \\
\hline
\end{tabular}


The importance of the Perciformes in the present study is consistent with the pattern found in northeastern Brazil estuaries (Teixeira \& Falcão 1992, Alves \& Soares Filho 1996, Santos 2000, Araújo et al. 2000, Paiva et al. 2008), Bahia (Oliveira-Silva et al. 2008, Reis-Filho et al. 2010) and with inventories of the coastal zone in Southern Bahia (Moraes et al. 2009). The large number of rare species and low abundant species found in this study is consistent with the diversity pattern in a tropical environment (Pauly \& Longhurst 2007). This theory can be applied in tropical estuaries where salinity fluctuations and related phenomena would also be responsible for the abundance and distribution of fish species (Vieira \& Music 1993). Tropical regions are less affected by climate changing, thus there is bigger specialization of various species, favoring those with high probability of extinction to persist, resulting in a large number of rare species in the community (Giller 1984).

Evaluating the shallow areas with high prevalence of muddy sediment, there was numerical dominance of Gobiidae, Gerreidae and Tetraodontidae, however it does not fully corroborate (due to Gobiidae) with the estuaries in Southern Brazil (Corrêa 2000, Ramos \& Vieira 2001, Schwarz Jr. 2005, Gomes 2005), estuary from northeast using seine net (Paiva et al. 2008) and from north utilizing block nets in mangrove tidal creeck (Barletta et al. 2003). On sandy beaches in the Todos os Santos Bay near the Paraguaçu River estuary, OliveiraSilva et al (2008) also using seine net it was not found Gobiidae and Tetraodontidae with high abundance. Yet, Reis-Filho et al. (2010), using the same method from the authors above found Gobiidae among the four most abundant families in semi-urban estuary on the north coast of Bahia, configuring the study of estuarine fish closer to the Paraguaçu River estuary (about $220 \mathrm{~km}$ ). But due to the acute environmental degradation (e.g. urbanization, removal of vegetation, pollution) that this system presents, comparisons of its ichthyofauna with least impacted estuaries should be made carefully.

The bottom nets accessed the ichthyofauna from the main channel dominated by Ariidae and Sciaenidae around all the Paraguaçu channel (lower estuary), similar pattern to Caeté River estuary,
Northern Brazil (Barletta et al. 2005), Sepetiba Bay, South-East Brazil (Azevedo et al. 2002) and Paranaguá estuary, Southern Brazil (Barletta et al. 2008). Although, in the middle estuary, using bottom nets, the dominant family was Sciaenidae with Stelifer rastrifer and Cynoscion microlepidotus showing higher catches and greater constancy of occurrence. Barletta et al. (2005) found S. rastrifer in the three sectors from Caeté River estuary (lower, middle and upper). However, in the present study this species occurred only in the middle and upper estuary preferring less saline waters. Still evaluating the shallow regions, gerreids appear as abundant and constant in the three sectors from the Formoso River estuary, Pernambuco (Paiva et al. 2008). In these last study and in estuary of the south (Falcão et al. 2006), clupeiform were abundantly collected, this group was among the most abundant on beaches near the study area (Oliveira Silva et al. 2008). In the present study, more clupeiform were caught with surface and bottom gill $(5-10 \mathrm{~m})$ in the middle estuary. Indeed, the dominance of gobies in shallow areas is a peculiar pattern in the structure of fish from the Paraguaçu River estuary.

There is a significant contribution of marine species in the inner estuary, most notably in mangrove creeks (Castro 2001) during a certain stage of the life cycle where the juveniles use these environments (Barletta et al. 2003, Oliveira-Neto et al. 2008). However, was observed an advancement of marine species, especially the adults (marine stragglers, marine migrants) for inner regions toward the middle estuary, showing greatest abundance and constancy of occurrence in this sector (e.g. Caranx latus Agassiz, 1831, Chloroscombrus chrysurus (Linnaeus, 1766), Polydactylus virginicus (Linnaeus, 1758), Pellona harroweri, Harengula clupeola (Cuvier, 1829), Opisthonema oglinum (Lesueur, 1818) and adults of Macrodon ancylodon (Bloch \& Schneider, 1801) and Isopistus parvipinis (Cuvier, 1830)). It's accepted that this movement toward middle estuary may be due to the increased salinity in this region, and in turn the estuary did not show the three zones (upper, middle and lower) with salinity gradient set for estuaries of Brazil as shown by Barletta et al. $(2005,2008)$ evaluating these saline conditions. The

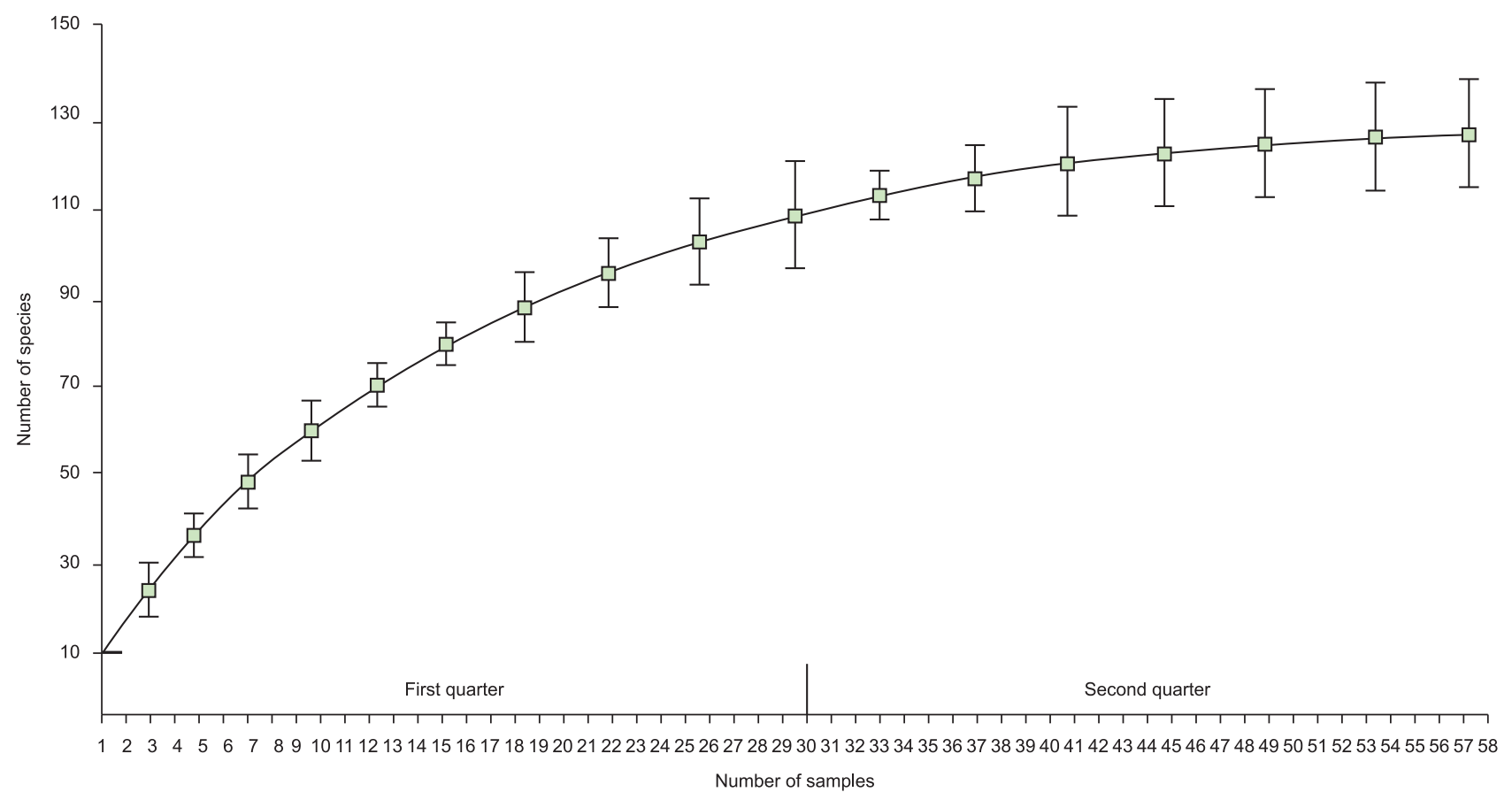

Figure 3. Species accumulation curve of the Paraguaçu River estuary by technique of rarefaction for $1 / 2$ of the samples from the first and second quarter.

Figura 3. Curva de acumulação de espécies para o estuário do Rio Paraguaçu pela técnica de rarefação para $1 / 2$ das amostras do primeiro e segundo trimestres. 

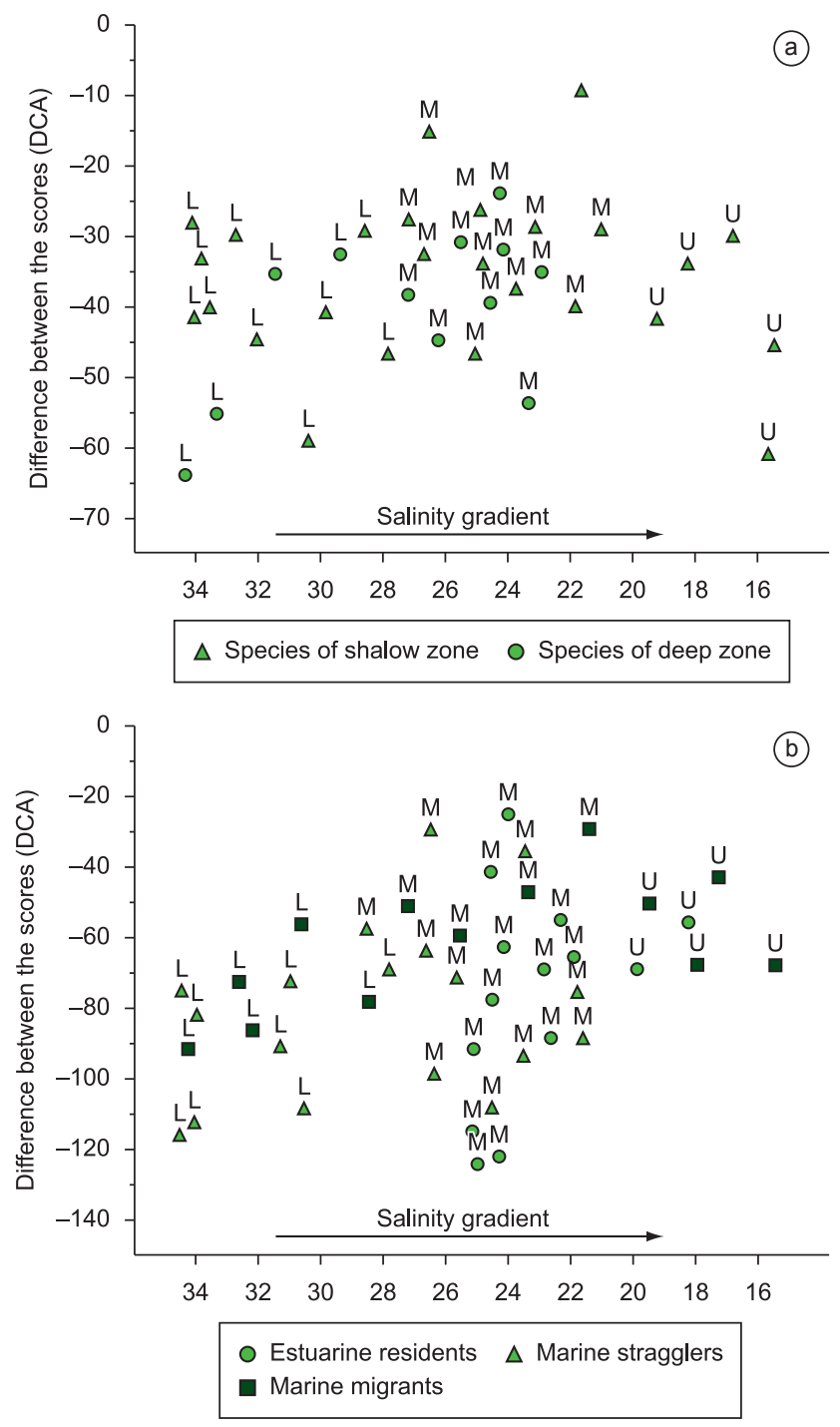

Figure 4. Scores of species derived from DCA obtained for the Paraguaçu River estuary. A: For depth zones. B: For movement of species in the estuary. ( $\mathrm{L}=$ lower estuary, $\mathrm{M}=$ middle estuary; $\mathrm{U}=$ upper estuary).

Figura 4. Escores das espécies derivadas da DCA obtida para o estuário do Rio Paraguaçu. A: Para zonas de profundidades. B: Para o movimento das espécies no estuário. ( $\mathrm{L}$ = baixo estuário, $\mathrm{M}=$ meio estuário; $\mathrm{U}=$ alto estuário).

ichthyofauna showed changes in distribution and structure that may be consequence of the hydrological regime imposed by the Pedra do Cavalo Dam. However, characteristics of this estuary fish community seem to be peculiar to that system as dominance of gobies in the shallow areas and Sciaenidae in middle estuary. We supported here a joint assessment of factors that influence the distribution of species within the estuary like physical-chemical gradients (Maes et al. 1998) and depth (Hyndes et al. 1999) besides those already studied as tolerance to salinity (Marshall \& Elliot 1998, Barletta et al. 2005), temperature (Peterson \& Ross 1991, Jaureguizar et al. 2003, Jaureguizar et al. 2004); and turbidity (Cyrus \& Blaber 1992) should be incorporated into studies aiming to understand the ichthyofaunistic patterns of the Brazilian estuaries.

\section{Acknowledgments}

We thank Harrys Jonhoson (EUA) by revision in english, the Coordenadoria de Aperfeiçoamento de Pessoal de Nível Superior
(CAPES) provided scholarships to J.A.R-F and the Conselho Nacional de Desenvolvimento Científico e Tecnológico (CNPq) provided scholarships to J.A.C.C.N. The present study was conducted with the authorization $n^{\circ}$ 21581-7 granted by Instituto Chico Mendes de Conservação da Biodiversidade (ICMBio). We also thank the anonymous referees for their thoughtful comments.

\section{References}

ALBUQUERQUE, P.G. 1994. Recursos potenciais de peixes da fauna acompanhante da pesca de camarões da foz do rio São Francisco - Litoral Norte, Piaçabuçu - AL. Dissertação de Mestrado, Universidade Federal de Pernambuco, 89p.

ALVES, M.I.M. \& SOARES FILHO, A.A. 1996. Peixes do estuário do Rio Jauaribe (Ceará-Brasil): Aspectos Fisioecolóicos. Ciên. Agron. 27(1):1-16

ANDRADE-TUBINO, M.F., RIBEIRO, A.N.R. \& VIANA, M. 2008. Organização espaço-temporal das ictiocenoses demersais nos ecossistemas estuarinos brasileiros: Uma síntese. Oecol. Bras. 12(4):640-661.

ARAÚJO, M.E., TEIXEIRA, J.M.C. \& OLIVEIRA, A.M.E. 2000. Ictiofauna marinha do estado do Ceará, Brasil, III. Actinopterygii de estuários. Arq. Ciênc. Mar. 33:139-142.

AZEVEDO, M.C.C., ARAÚJO, F.G., PESSANHA, A.L.M. \& SILVA, M.A. 2002. Co-occurrence of demersal fishes in a tropical bay in southeastern Brazil: a null model analysis. Est., Coas. Shelf. Scie. 66:315-322.

BARLETTA, M. \& COSTA, M.F. 2009. Living and non-living resources exploitation in a tropical semi-arid estuary. Jour. Coast. Resea. 56:371375.

BARLETTA, M., AMARAL, C.S., CORRÊA, M.F.M., GUEBERT, F., DANTAS, D.V., LORENZI, L. \& SAINT-PAUL, U. 2008. Factors affecting seasonal variations in demersal fish assemblages at an ecocline a tropical-subtropical estuary. Jour. Fish. Biol. 73:1314-1336.

BARLETTA, M., BARLETTA-BERGAN, A., SAINT-PAUL, U. \& HUBOLD, G. 2003. Seasonal changes in density, biomass, and diversity of estuarine fishes in tidal mangrove creeks of the lower Caeté Estuary (northern Brazilian coast, east Amazon). Mar. Ecol. Prog. Ser. 256:217-228.

BARLETTA, M., BARLETTA-BERGAN, A., SAINT-PAUL, U. \& HUBOLD, G. 2005. The role of salinity in structuring the fish assemblages in a tropical estuary. Jour. Fish. Biol. 66:45-72.

BARLETTA-BERGAN, A. 1999. Structure and seasonal dynamics of larval and juvenile fish in the mangrove-fringed estuary of the Rio Caeté in North Brazil. Zentrum fur Marine Tropenokologie, 220p. Contribution 8.

BARROS, A.C. \& JONSSON, S. 1967. Prospecção de camarões na região estuarina do rio São Francisco. Bol. Est. Pes. 7(2):7-29.

BARROS, F., HATJE, V., FIGUEIREDO, M.B., MAGALHÃES, W.F., DÓREA, H.S. \& EMÍDIO, E.S. 2008. The structure of the benthic macrofaunal assemblages and sediments characteristics of the Paraguaçu estuarine system, NE, Brazil. Est. Coast. Shelf. Scie. 78:758-762.

BEAUMORD, A C. 1991. As comunidades de peixes do rio Manso.Chapada dos Guimarães, MT: uma abordagem ecológica numérica. Dissertação de Mestrado, Instituto de Biociências Carlos Chagas, 108p.

BLABER, S.J.M. \& BLABER, T.G. 1980. Factor affecting the distribution of juvenile estuarine and inshore fish. Jour. Fish. Biol. 17:143-162.

CAMARGO, M. 1999. Biologia e estrutura populacional das espécies da família Scianidae (Pisces: Perciformes), no estuário do rio Caeté, Município de Bragança, Pará-Brasil. Dissertação de Mestrado, Museu Paraense Emílio Goeldi, Universidade Federal do Pará, 84p.

CARPENTER, K.E. 2002a. The living marine resources of the Western Central Atlantic. In FAO Species Identification Guide for Fishery Purposes and American Society of Ichthyologists and Herpetologists. FAO, Rome, v.2, part 1, p.601-1374. Special publication, 5.

CARPENTER, K.E. 2002b. The living marine resources of the Western Central Atlantic. In FAO Species Identification Guide for Fishery Purposes and American Society of Ichthyologists and Herpetologists. FAO, Rome, v.3, part 2, p.1375-2127. Special publication, 5. 
CARVALHO NETA, R.N.F. \& CASTRO, A.C.L. 2008. Diversidade das assembléias de peixes estuarinos da Ilha dos Caranguejos, Maranhão. Arq. Ciên. Mar. 41:48-57.

CARVALHO, J.B, 2000. Caracterização morfoestratigráfica do preenchimento sedimentar da Baía de Iguape, Bahia - Influência das variações eustáticas do nível do mar e atividades tectônicas recentes. Dissertação de Mestrado, Universidade Federal da Bahia, 119p.

CASATti, L., CASTRO, R.M.C. \& LANGEANI, F. 2001. Peixes de riacho do Parque Estadual Morro do Diabo, bacia do alto rio Paraná, SP. Biota Neotrop. 1(1): http://www.biotaneotropica.org.br/v1n12/pt/ fullpaper?bn00201122001+pt (último acesso em 22/03/2010).

CASTRO, A.C.L. 2001. Diversidade da assembléia de peixes em iarapés do estuário do Rio Paciência (MA-Brasil). Atlântica. 23:39-46.

CORRÊA, M.F.M. 2000. Ictiofauna demersal da Baía de Guaraqueçaba (PARANÁ, BRASIL). Composição, estrutura, distribuição espacial, variabilidade temporal e importância como recurso. Tese de Doutorado em Zoologia, Setor de Ciências Biológicas, Universidade Federal do Paraná, 160p.

CYRUS, D.P. \& BLABER, S.J. M. 1992. Turbidity and salinity in a tropical Northern Autralian estuary and their influence on fish distribution. Est. Coast. Shelf. Scien. 35:545-563.

DAJOZ, R. 1973. Ecologia geral. 2. ed. Ed. Vozes/EDUSP, São Paulo.

DAY, J.W., HALL, C.A.S., KEMP, W.M. \& YAÑEZ-ARANCIBIA, A. 1989. Estuarine Ecology. Wiley, New York.

ELLIOTT, M., WHITFIELD, A.K., POTTER, I.C., BLABER, S.J.M., CYRUS, D.P., NORDLIE, F.G. \& HARRISON, T.D. 2007. The guild approach to categorizing estuarine fish assemblages: a global review. Fish and Fisheries. 8:241-268.

EMPRESA BAIANA DE PESCA - BAHIAPESCA. 1998. Boletim estatístico de pesca estuarina e marinha no Estado da Bahia. Programa de desenvolvimento da aqüicultura e da pesca. Governo da Bahia.

ESCHMEYER, W.N. 2006. Catalog of Fishes. California Academy of Sciences, San Francisco.

ESKINAZI, A.M. 1970. Lista preliminar dos peixes estuarinos de Pernambuco e Estados Vizinhos (Brasil). Trab. Ocean. Uni. Fed. Pe. 9:265-274.

ESKINAZI, A.M. 1972. Peixes do Canal de Santa Cruz-Pernambuco-Brasil. Trab. Ocean. Uni. Fed. Pe. 13:283-302.

FALCÃO, M.G., SARPÉDONTI, H.L., SPACH, H.L., OTERO, M.E.B., QUEIROZ, G.M.L.N. \& SANTOS, C.A. 2006. Ictiofauna em planícies de maré das Baías das Laranjeiras e de Paranaguá, Paraná, Brasil. Rev. Bras. Zoo. 8(2):125-138.

FAUNCE, C.H. \& SERAFY, J.E. 2006. Mangroves as fish habitat: 50 years of field studies. Mar. Ecol. Prog. Ser. 318:1-18.

FIGUEIREDO, J.L. \& MENEZES N.A. 1978. Manual dos peixes marinhos do Sudeste do Brasil. II. Teleostei (1). Universidade de São Paulo, São Paulo.

FIGUEIREDO, J.L. \& MENEZES N.A. 1980. Manual dos peixes marinhos do Sudeste do Brasil. III. Teleostei (2). Universidade de São Paulo, São Paulo.

FIGUEIREDO, J.L. \& MENEZES N.A. 2000. Manual dos peixes marinhos do Sudeste do Brasil. VI. Teleostei (5). Universidade de São Paulo, São Paulo.

FISHER, L.G., PEREIRA, L.E.D. \& VIEIRA, J. 2004. Peixes estuarinos e costeiros. Ecoscientia, Rio Grande, 139p. Série Biodiversidade do Atlântico Sudoeste.

FROESE, R. \& PAULY, D. 2006. FISHBASE. www.fishbase.org/home.htm (último acesso em 03/09/2009).

GENZ, F., LESSA, G.C. \& CIRANO, M. 2008. Vazão mínima para estuários: um estudo de caso do Rio Paraguaçu/BA. Rev. Bras. Rec. Hid. 13:73-82.

GILLER, P.S. 1984. Community structure and the niche. Chapman and Hall, London.
GODEFROID, R.S., SPACH, H.L., SANTOS, C., MACLAREN, G. \& SCHWARZ Jr., R. 2004. Mudanças temporais na abundância e diversidade da fauna de peixes do infralitoral raso de uma praia, sul do Brasil. Iheringia Sér. Zoo. 94(1):95-104.

GOMES, I.D.A. 2005. Estrutura da ictiofauna demersal do Paraná, entre os sistemas de Baía de Guaratuba e a Foz do Rio Saí-Guaçu. Tese de Doutorado em Zoologia. Setor de Ciências Biológicas, Universidade Federal do Paraná, 113p.

HYNDES, G.A., POTTER, I.C., PLATELL, M.E. \& LENANTON, R.C.J. 1999. Does the composition of the demersal fish assemblages in temperate coastal waters change with depth and undergo consistent seasonal changes? Marine Biology. 134:335-352.

JAUREGUIZAR, A.J., MENNI, R., BREMEC, C., MIANZAN, H. \& LASTA, C. 2003. Fish assemblage and environmental patterns in the Río de La Plata estuary. Est. Coast. Shelf. Scien. 56:921- 933.

JAUREGUIZAR, A.J.; MENNI, R.; GUERRERO, R. \& LASTA, C. 2004. Environmental factors struturing fish communities of the Río de La Plata estuary. Fish. Res. 66:195-211.

MAES, J., VAN DAMME, P.A., TAILLIEU, A. \& OLLIVIER, F. 1998. Fish communities along na oxygen-poor salinity gradient (Zeeschelde Estuary, Belgium). Jour. Fish. Biol. 52:534-546.

MARCENIUK, A.P. \& MENEZES, N.A. 2007. Systematics of the family Ariidae (Ostariophysi, Siluriformes), with a redefinition of the genera. Zootaxa 1416:1-126.

MARCENIUK, A.P. 2005. Chave de identificação das espécies de bagres marinhos (Siluriformes, Ariidae) da costa brasileira. B. Inst. Pesca. 31(2):89-101.

MARSHALL, S. \& ELLIOTT, M. 1998. Environmental influences on the fish assemblage of the Humber estuary. Est. Coast. Shelf. Scien. 46:175-184

MENEZES, N.A. \& FIGUEIREDO, J.L. 1980. Manual de peixes marinhos do Sudeste do Brasil. IV. Teleostei (3). Universidade de São Paulo, São Paulo.

MENEZES, N.A. \& FIGUEIREDO, J.L. 1985. Manual de peixes marinhos do Sudeste do Brasil. V. Teleostei (4). Universidade de São Paulo, São Paulo.

MENEZES, N.A., BUCKUP, P.A., FIGUEIREDO, J.L. \& MOURA, R.L. 2003. Catálogo das espécies de peixes marinhos do Brasil. Universidade de São Paulo, São Paulo.

MORAES, L.E., ROMERO, R.M., ROCHA, G.R.A. \& MOURA, R.L. 2009. Demersal ichthyofauna of the inner continental shelf off Ilhéus, Bahia, Brazil. Biota Neotrop. 9(4): http://www.biotaneotropica.org.br/v9n4/en/ abstract?inventory+bn01409042009.

MOURA, R.L. \& LINDEMAN, K.C. 2007. A new species of snaper (Perciformes: Lutjanidae) from Brazil, with comments on the distribution of Lutjans griseus and L. apodus. Zootaxa. 1422:31-43.

NELSON, J.S. 1994. Fish of the world. 3. ed. John Wiley \& Sons, New York.

OLIVEIRA, A.M.E. 1972. Peixes estuarinos do Nordeste Oriental Brasileiro. Arq. Cien. Mar. 12(1):35-41.

OLIVEIRA, A.M.E. 1974. Ictiofauna das águas estuarinas do Rio Parnaíba (Brasil). Arq. Cien. Mar. 14(1):41-45.

OLIVEIRA, A.M.E. 1976. Composição e distribuição da ictiofauna, nas águas estuarinas do Rio Jaguaribe (Ceará - Brasil). Arq. Cien. Mar. 16(1):9-18.

OLIVEIRA-NETO, J.F., SPACH, H.L., SCHWARZ-JUNIOR, R. \& PICHLER, H.A. 2008. Diel variaton in fish assemblages in tidal creeks in the southern Brazil. Braz. J. Biol. 68(1):37-43.

OLIVEIRA-SILVA, J.T., PESO-AGUIAR, M.C. \& LOPES, P.R. 2008. Ictiofauna das praias de Cabuçu e Berlinque: uma contribuição ao conhecimento das comunidades de peixes na Baía de Todos os Santos Bahia - Brasil. Biotemas 21(4):105-115.

PAIVA, A.C.G., CHAVES, P.T.C. \& ARAÚJO, M.E. 2008. Estrutura e organiação tróficada ictiofauna de ágas rasas em um estuário tropical. Rev. Bras. Zoo. 25:647-661.

PATERSON, A W. \& WHITFIELD, A.K. 2000. Do shallow water habitats function as refugia for juvenile fishes? Est. Coast. Shelf. Scien. 51:359-364. 
PAULY, D. \& LONGHURST, A.R. 2007. Ecologia dos Oceanos Tropicais. Edusp, São Paulo.

PETERSON, M. S. \& ROSS, S. T. 1991. Dynamics of littoral fishes and decapods along coastal river-estuarine gradient. Est. Coast. Shelf. Scien. 33:467-483.

RAMOS, L.A. \& VIEIRA, J.P. 2001. Composição específica e abundância de peixes de zonas rasas dos cinco estuários do Rio Grande do Sul, Brasil. Bol. Inst. Pesca. 27(1):109-121.

REIS-FILHO, J.A., CAVALCANTE, L.D.N., MENEZES, B.L. \& SOUZA, G.B.G. 2010. Variação espaço-temporal e efeito do ciclo lunar na ictiofauna estuarina: Evidências para o estuário do Rio Joanes - Bahia. Biotemas 23(2):111-122.

REIS-FILHO, J.A., BARROS, F., NUNES, J.A.C.C., SAMPAIO, C.L. \& SOUZA, G.B. In press. Moon and tide effects on fish capture in a tropical tidal flat. J. Mar. Biol. Assoc. UK.

SANTOS, A.C.A., OLIVEIRA-SILVA, J.T., MOURA, P.E.S. \& SENA, M.P. 2009. Ampliação do limite norte de distribuição geográfica de Catathyridium garmani (Jordan \& Goss 1889) (Actinopterygii: Achiridae). Biota Neotrop. 9(1): http://www.biotaneotropica.org.br/ v9n1/pt/abstract?short-communication+bn02409012009 (último acesso em 24/05/2010).
SANTOS, C., SCHWARZ Jr., R., OLIVEIRA NETO, J.F. \& SPACH, H.L. 2002. A ictiofauna em duas planícies de maré do setor euhalino da Baía de Paranaguá, PR. Bol. Inst. Pesc. 28(1):49-60.

SANTOS, M.C.F. 2000. Diversidade ecológica da ictiofauna acompanhante nas pescarias de camarões em Tamandaré (Pernambuco-Brasil). Bol. Téc. Cien. CEPENE. 8:7-27.

SANTOS, M.C.F., FREITAS, A.E.T.S. \& SILVA, M.M. 1998. Composição da ictiofauna acompanhante da pesca de camarão em Tamandaré/PE e Pontal do Peba/AL. Bol. Téc. Cien. CEPENE. 6:47-60.

SCHWARZ Jr., R. 2005. A ictiofauna demersal da Baía dos Pinheiros, Paraná. Curitiba, Dissertação de Mestrado em Zoologia, Setor de Ciências Biológicas, Universidade Federal do Paraná, 85p.

TEIXEIRA, R.L. \& FALCÃO, G.A.F. 1992. Composição da fauna nectônica do complexo lagunar Mundáu/Manguaba, Maceió-AL. Atlântica 4:4358.

VIEIRA, J.P. \& MUSIC, J.A. 1993. Latitudinal patterns in diversity os fishes in warm temperature and tropical estuarine waters of the western Atlantic. Atlântica 15:115-133.

WHITFIELD, A.K. 1999. Ichthyofaunal assemblages in estuaries: a South African case study. Revie. Fish. Biol. Fishe. 9:151-186. 
